\title{
Rastreo de cáncer de colon con sangre oculta en materia fecal Técnicas sugeridas para su uso e interpretación
}

Suggested technique for fecal occult blood testing and interpretation in colorectal cancer screening

American College of Physicians. Ann Intern Med 1997;126:808:810 Screening for Colorectal Cancer with the Fecal Occult Blood Test: A background paper. D.Ransohoff, C.Lang Ann Intern Med 1997; 126:811:822

\section{Objetivo}

Proveer información para realizar rastreo de cáncer de colon con sangre oculta en materia fecal (SOMF), interpretar los resultados y planear el manejo de los pacientes.

\section{Diseño}

Revisión de la literatura sobre rastreo y validación de los tests de SOMF
Fuente de los datos

MEDLINE entre 1984 y 1996.

Selección de estudios

Evaluación subjetiva de los autores

Datos principales

Tres ensayos clínicos randomizados demostraron reducir la mortalidad por cáncer colorectal mediante el rastreo con SOMF.

Según reflejan sus diseños, no hay consenso acerca de la frecuencia del rastreo, conveniencia de restricciones dietéticas previas, rehidratación de la muestra y evaluación de los pacientes con resultados positivos.

Tabla 1: Principales estudios randomizados finalizados de SOMF como rastreo de cáncer colorrectal

\begin{tabular}{|c|c|c|c|}
\hline & Minnesota (1) & Gran Bretaña (2) & Dinamarca (3) \\
\hline Seguimiento & 13 años & 7.8 años & 10 años \\
\hline Participantes & 46.551 & 152.850 & 61.933 \\
\hline Muestra & Voluntaria & Poblacional & Poblacional \\
\hline Frecuencia & Anual & Cada 2 años & Cada 2 años \\
\hline Rehidratación de la muestra & $\mathrm{Si}$ & No & No \\
\hline Adherencia $(\%)$ & $\begin{array}{c}\text { ler examen } 90.2 \% \\
\text { Completa } 46.2 \\
\end{array}$ & $\begin{array}{c}\text { 1er examen } 59.6 \% \\
\text { Completa } 38.2 \\
\end{array}$ & $67 \%$ \\
\hline $\begin{array}{l}\text { Posibilidad de encontrar un cáncer } \\
\text { colorectal temprano luego de SOMF } \\
\text { positiva. }\end{array}$ & No aclarado & Inicial: $7 \%$ & $\begin{array}{l}\text { inicial: } 14 \% \\
\text { repetición: } 6.3 \%\end{array}$ \\
\hline $\begin{array}{l}\text { Reducción absoluta de la mortalidad } \\
\text { global }\end{array}$ & $0.295 \%$ & No hubo reducción & $0.31 \%$ \\
\hline $\mathrm{NNR}^{*}$ & 337 & - & 333 \\
\hline
\end{tabular}

* Número Necesario a Rastrear: Cantidad de pacientes que deben ser rastreados durante ese período para salvar una vida.

\section{Tipos de pruebas para SOMF}

Las ventajas de los tests de guayaco como el Hemoccult II y HemoccultSENSA es que son baratos y pueden ser hechos en el consultorio. Los tests inmunoquímicos como el HemeSelect y HemoQuant son más específicos, pero deben ser hechos en el laboratorio y son más caros.

\section{Conclusión}

La IS fue un preddictor independiente de muerte de causa coronaria, si bien el pronóstico de estos pacientes fue mejor que el de otras formas clínicas de enfermedad coronaria.

\section{Sensibilidad y especificidad*}

El grado de la reducción de la mortalidad por cáncer de colon depende de la sensibilidad de los tests de SOMF. Como éstos tienen resultados falsos negativos, la reducción de la mortalidad no puede ser óptima. Por este motivo los médicos no deberían considerar que los pacientes con resultados negativos del test se en- cuentran protegidos de la enfermedad. Por lo tanto debería ser evaluado el colon en todo paciente con signos o síntomas sospechosos, aún con SOMF negativa.

Para calcular la sensibilidad de la SOMF se definió al cáncer como al tumor diagnosticado dentro del año de la realización de la misma. La sensibilidad de la SOMF para la detección de pacientes con cáncer curable (Dukes A o B) se estima entre el 30 y $50 \%$.

La especificidad de la SOMF es el principal determinante de costo del rastreo ya que la mayoría de las colonoscopías que se hicieron, fueron a consecuencia de resultados falsos positivos. La tasa de falsos positivos es del $2-4 \%$ con muestras sin rehidratación y de más del $8 \%$ con rehidratación.

En los pacientes con SOMF positiva hecha con muestra no rehidratada, la probabilidad de encontrar un cáncer colorectal temprano (potencialmente curable) o adenomas grandes fue del 17 a $46 \%$ (Valor Predictivo Positivo *). Por lo tanto estos pacientes merecen una evaluación colorectal completa. 
Evaluación de pacientes con SOMF positiva

Siempre que sea posible, debe hacerse una fibrocolonoscopía, ya que permite resecar en un único procedimiento los pólipos que pudieran ser hallados durante la misma. Sin embargo es más cara, requiere sedación y tiene mayor riesgo de perforación.

Otra opción es realizar una rectosigmoideoscopía junto con estudios radiográficos de colon por enema con doble contraste. Esta opción es más barata, tiene menos complicaciones y es la preferida en los pacientes con adherencias pélvicas, en aquellos que no puedan suspender un tratamiento anticoagulante o en quienes la sedación sea riesgosa. Sin embargo tiene una sensibilidad de sólo el $60 \%$ para detectar adenomas mayores de $1 \mathrm{~cm}$ y $15 \%$ de los pacientes requieren fibrocolonoscopía subsecuente por resultados dudosos.

Luego de una fibrocolonoscopía negativa podría suspenderse el rastreo con SOMF por 5 años.

El costo por año de vida salvado ha sido estimado en 20.000 dólares.

Tabla 2.Recomendaciones

\begin{tabular}{|l|c|}
\hline \multicolumn{1}{|c|}{ Recomendación } & $\begin{array}{c}\text { Calidad de la } \\
\text { recomendación }\end{array}$ \\
\hline $\begin{array}{l}\text { Se podría comenzar el rastreo con SOMF desde los 55 o } 60 \\
\text { años. }\end{array}$ & Débil \\
\hline $\begin{array}{l}\text { Usar Hemocult II con } 3 \text { deposiciones diferentes (2 muestras } \\
\text { cada una). No debe usarse como muestra los restos de materia fecal obtenidos } \\
\text { mediante tacto rectal. }\end{array}$ & Fuerte \\
\hline Restringir el uso de antiinflamatorios no esteroideos. & Débil \\
\hline $\begin{array}{l}\text { Sugerir a los pacientes que no consuman carnes rojas, pescados, } \\
\text { aves, vitamina C y verduras crudas. }\end{array}$ & Débil \\
\hline $\begin{array}{l}\text { Procesar las muestras dentro de la semana de haber sido } \\
\text { obtenidas. }\end{array}$ & Moderada \\
\hline $\begin{array}{l}\text { Evaluación completa del colon si SOMF es positiva } \\
\text { Fibrocolonoscopía o Sigmoideoscopia con Colon por enema (preferiblemente } \\
\text { doble contraste) }\end{array}$ & Débil \\
\hline $\begin{array}{l}\text { Evaluar el colon completo dentro de 2-3 meses a los pacientes } \\
\text { con SOMF positiva. }\end{array}$ & Moderada \\
\hline Frecuencia anual o bianual & Moderada \\
\hline La muestra no debe ser rehidratada & Moderada \\
\hline $\begin{array}{l}\text { Considerar test positivo si al menos 1 de las } 6 \text { muestras es } \\
\text { positiva }\end{array}$ & \\
\hline
\end{tabular}

*RR: Riesgo relativo, ver glosario.

\section{Comentario}

Tres ensayos clínicos randomizados y controlados demostraron reducción de la mortalidad por cáncer colorectal mediante el rastreo con SOMF. Sin embargo sólo dos de ellos demostraron reducir la mortalidad total (Ver tabla).

La adherencia al rastreo fue mayor en el trabajo de Minnesota (participantes voluntarios), que en los que usaron muestras poblacionales, siendo éste un claro ejemplo de que una intervención puede tener mejor resultado en el escenario favorable de un trabajo controlado (eficacia*) y menor en las condiciones reales de la práctica cotidiana (efectividad*). La presente guía se ocupa de revisar las distintas técnicas utilizadas para investigar la presencia de SOMF y algunas sugerencias para su interpretación. Llama la aten- ción que el grado de las recomendaciones se presentan como "débil o fuerte" en lugar de los grados A, B y C más ampliamente aceptados *, pudiendo esto llevar a confusión.

En cuanto a la conveniencia de rehidratar o no la muestra, cada comunidad deberá decidir de acuerdo a sus costos, realidad geográfica y prioridades cual estrategia es la más adecuada a su ámbito. La rehidratación trae como consecuencia el aumento de la sensibilidad del test y la disminución de la especificidad, pudiendo multiplicar hasta por cuatro la tasa de falsos positivos. Sin embargo, la rehidratación puede ser necesaria si se demora el procesamiento de la muestra; esto evitaría la disminución de la sensibilidad por el secado de la misma. Este problema podría ser mi- 
nimizado si el test fuera hecho en los consultorios.

Cabe aclarar que en caso de indicarse rastreo debería realizarse con tests en base a guayaco y no con bencidina, muy difundida en nuestro medio, ya que estos resultados provienen de trabajos hechos en base al test de guayaco.

Con respecto a la estimación de la sensibilidad de la SOMF, es de destacar que no se realizó en base a un test de referencia (no todos los pacientes hicieron SOMF y fibrocolonoscopía) sino que se consideró como referencia el diagnóstico de cáncer de colon dentro del año de realizada la SOMF, periodo que puede resultar insuficiente para la manifestación clínica de la enfermedad.

En resumen, el rastreo del cáncer colorectal con SOMF demostró ser una estrategia adecuada para reducir la mortalidad por cáncer de colon en tres ensayos clínicos randomizados y controlados y reducir la mortalidad general en dos de ellos.

A la hora de decidir la instrumentación de políticas de salud debemos recordar que un paciente rastreado con SOMF anual durante 10 años tiene más de $40 \%$ de probabilidad de tener un falso positivo a lo largo del periodo y, por ende, necesitar una fibrocolonoscopía. A pesar de que el costo de la SOMF es baio, hay que tener en cuenta que el rastreo es una estrategia poblacional que determina la realización de un gran número de colonoscopías, es- tudio de alto costo ( $\$ 300$ en Argentina), con efectos adversos no despreciables ( 2 perforaciones $/ 1000$ procedimientos), y que requiere personal calificado.

Cada sistema de salud deberá decidir sus prioridades sanitarias analizando los costos que pueda afrontar y el grado de aceptación de este tipo de rastreo tanto en la población, como en la comunidad médica.

*Ver glosario

Dr Sergio Terrasa

Unidad de Medicina Familiar y Preventiva Hospital Italiano de Buenos Aires

\section{Referencias}

Mandel J, Bond J, Church T et al. Reducing mortality from clorectal cancer by screening for faecal occult blood. Minnesota Colon Cancer Control Study. N Engl J Med. 1993;328:1365-1371

Kronborg O, Fenger C, Olsen $\mathrm{J}$ et al. Randomised study of screening for colorectal cancer with faecal occult blood test. Lancet 1996;348:1467-147 Hardcastle J, Chamberlain, Robinson M et al. Randomised controled trial of faecal-occult blood screening for colorectal cancer. Lancet 1996;348:1472-1477 